\title{
Frequency of Hypoglycaemia after Different Bariatric Surgical Procedures
}

\author{
Johanna Maria Brix ${ }^{a, b}$ Hans-Peter Koppa, ${ }^{a}$ Florian Höllerla, b \\ Gerit Holger Schernthaner $^{c}$ Bernhard Ludvik ${ }^{a}$ b Guntram Schernthaner ${ }^{a}$ \\ a Department of Medicine I, Rudolfstiftung Hospital Vienna, Vienna, Austria; \\ ${ }^{b}$ Karl Landsteiner Institute for Obesity and Metabolism, Vienna, Austria; ' ${ }^{\text {Division of }}$ \\ Angiology, Department of Medicine II, Medical University of Vienna, Vienna, Austria
}

\section{Keywords}

Hypoglycaemia $\cdot$ Bariatric surgery $\cdot$ Morbid obesity

\begin{abstract}
Objectives: The frequency of postprandial hypoglycaemia after different operative procedures of bariatric surgery (BS) is unknown, although this complication is potentially dangerous. Predictors and severity of hypoglycaemia after Roux-en-Y gastric bypass (RYGB), sleeve gastrectomy, and gastric banding were investigated in a large prospective study. Methods: This study was performed at an excellence centre for BS at a tertiary care institution. Data of 333 patients (mean BMl: $44.9 \pm 9.6 \mathrm{~kg} / \mathrm{m}^{2}$; mean age: $40 \pm 10$ years; $80.7 \%$ women) were analysed in a prospective study with a 2-year observation period after BS. All patients underwent a 2-hour oral glucose tolerance test (OGTT) with measurements of blood glucose (BG) and insulin. For the purpose of this study, hypoglycaemia was defined as a post-challenge BG $<2.8 \mathrm{mmol} / \mathrm{L}$ during the OGTT. Results: 72 (25.6\%) of 281 patients showed post-challenge hypoglycaemia after surgery. Hypoglycaemia was different after various procedures: $32.6 \%$ of patients after RYGB, 22.6\% after sleeve gastrectomy, but only $2.3 \%$ after gastric banding had hypoglycaemia. In the whole group, patients with hypoglycaemia had lost more weight $(p=0.013)$, had a slightly greater decrease in $\mathrm{BMI}(p=0.037)$, a greater change in 2 -hour postchallenge BG $(p=0.001)$, and a smaller change in 1-hour post-challenge insulin $(p=0.004)$ compared to patients without hypoglycaemia. Conclusion: This prospective study shows a higher prevalence of severe hypoglycaemia (25.6\%) after BS than anticipated from retrospective registers. A systematic evaluation of glucose and insulin levels by OGTT 2 years postsurgery may help to identify patients at increased risk for symptomatic and asymptomatic hypoglycaemia.


Brix et al.: Hypoglycaemia after Bariatric Surgery

\section{Introduction}

Bariatric surgery (BS) is now a common and effective treatment for patients with morbid obesity [1]. In a Swedish registry by Marsk and colleagues [2], patients had a two-fold to seven-fold relative risk for developing severe hypoglycaemia after gastric bypass surgery, although the absolute risk might be estimated low with a reported prevalence of $0.2 \%$. However, register studies succumb to their inherent methodological problems - especially bias regarding reported and unreported events. In addition, the frequency of hypoglycaemia after bariatric procedures, which are regarded as being more modern, such as sleeve gastrectomy, is not well known [3].

$\mathrm{BS}$ is a recognised method to treat type 2 diabetes in morbidly obese patients [4]. It seems logical to associate post-surgery hypoglycaemia with type 2 diabetes, but large studies have demonstrated that hypoglycaemia after BS occurs independently from the preoperative diabetic state [2].

The increase in publications on post-gastric bypass hypoglycaemia cases following the initial 2005 report, together with the increasing use of BS, suggests that this complication may not be rare $[3,5]$. Goldfine et al. [6] raised the suspicion that the frequency of asymptomatic and symptomatic hypoglycaemia after BS might be up to $30 \%$. In addition, the wide range in severity underscores the critical need to gain better knowledge of the true incidence of this complication $[6,7]$.

Therefore, we conducted a large longitudinal prospective study in patients with morbid obesity before and after different procedures of BS to investigate the individual frequency of hypoglycaemia.

\section{Methods}

\section{Study Design and Patients}

A total of 333 patients who fulfilled the criteria for BS were admitted to the outpatients department for obesity and consented to take part in a follow-up trial. Due to a strict followup regime (minimum two visits annually), only 15.6\% $(n=52)$ of the patients were lost during follow-up. Thus, 281 patients with morbid obesity (mean BMI: $44.9 \pm 9.6 \mathrm{~kg} / \mathrm{m}^{2}$; mean age: $40 \pm 10$ years; $80.7 \%$ women) were available with complete data for analysis and were investigated before and 2 years after BS. $62.3 \%(n=175)$ of the patients underwent laparoscopic Roux-en-Y gastric bypass surgery (RYGB), 22.1\% $(n=62)$ had a sleeve gastrectomy, and $15.6 \%(n=44)$ gastric banding. Surgery had been indicated according to the guidelines of the National Institutes of Health consensus statement for surgery in severe obesity (1992). Patients were systematically referred to a multidisciplinary team for medical, psychological, nutritional, and surgical expertise at the Outpatients Unit for Morbid Obesity of Rudolfstiftung Hospital, Vienna. Exclusion criteria for operation were as described previously [8]. In addition, patients with known diabetes before BS were not eligible. All patients attended the postoperative care follow-up programme organised by the multidisciplinary team.

\section{Analyses}

Anthropometric measurements as well as dietary and exercise habits were taken from all patients. Blood samples were taken after an overnight fast. All patients underwent a 2-hour 75-gram oral glucose tolerance test (OGTT) to evaluate post-challenge hypoglycaemia and prevalence of pre-/diabetes pre- and post-surgery. Blood glucose was measured by enzymatic in vitro tests (Roche Diagnostics GmbH, Graz, Austria). Insulin levels were measured by 
Brix et al.: Hypoglycaemia after Bariatric Surgery

chemiluminescence immunoassay. Glycosylated haemoglobin $\left(\mathrm{HbA}_{1 \mathrm{c}}\right)$ was measured by high-performance liquid chromatography (Diamat, Bio-Rad, Hercules, CA, USA).

\section{Symptoms of Hypoglycaemia}

Before OGTT, a questionnaire was handed out to the patients with typical symptoms of hypoglycaemia, such as sweating, dizziness, weakness or exhaustion, a general feeling of sickness, nausea, and hunger. Since no questionnaire for hypoglycaemia in patients without diabetes is validated and available in German, we developed our own questionnaire based on the knowledge and experience of all authors. A translated version can be found in the online appendix (for all online suppl. material, see www.karger.com/doi/10.1159/000493735). During the OGTT, the patients were asked to report their symptoms, if present. Patients were encouraged to ask the medical staff in case of not understanding a question. Therefore, the return rate was $92 \%$. In addition, medical staff neither participating in the study nor in the analysis was available for the patients to discuss the symptoms for clarification.

\section{Mixed Meal Tolerance Test}

To validate our results obtained from the OGTT, we randomly selected a subset of 14 patients, whose parameters did not differ from the total study cohort. In those patients, our conventional 2-hour OGTT as well as a 2-hour mixed meal tolerance test (MMTT) were performed. On the first day, an OGTT was performed and was followed after a 1-day break by a 2-hour MMTT. For the MMTT, we used one bottle of Ensure plus ${ }^{\circledR}$ (Abbott, Abbott Park, IL, USA) for each patient.

\section{Calculations and Statistics}

Hypoglycaemia was defined as a blood sugar $<2.8 \mathrm{mmol} / \mathrm{L}$ during the OGTT [9]. Homeostasis Model Assessment of Insulin Resistance (HOMA-IR) was calculated as described previously [10]. Insulin was converted from units of $\mu \mathrm{U} / \mathrm{mL}$ to $\mathrm{pmol} / \mathrm{L}$ using the conversion factor 6.945 , and glucose was converted from units of $\mathrm{mg} / \mathrm{dL}$ to $\mathrm{mmol} / \mathrm{L}$ by dividing by 18 where required when calculating the insulin secretory and insulin sensitivity indices. Insulinogenic index (IGI), defined as the ratio of difference in fasting to $60-\mathrm{min}$ insulin to glucose values $=$ $\left(\Delta \mathrm{I}_{0-60} / \Delta \mathrm{G}_{0-60}\right)$ as well as 120-min insulin to glucose values $=\left(\Delta \mathrm{I}_{0-120} / \Delta \mathrm{G}_{0-120}\right)[11]$. Data are shown as median (25th-75th percentile). Differences were analysed by Mann-Whitney, Wilcoxon, or Kruskal-Wallis test as appropriate. An $\alpha$ level of $p<0.05$ was considered significant. Statistical analyses were performed with SPSS 22.0 (IBM Corporation, Armonk, NY, USA).

\section{Results}

Two years after BS, in the whole cohort, weight had decreased by $31 \%$ of the total body weight and BMI decreased by $30 \%$. Risk markers such as blood pressure, glucose, and insulin levels decreased significantly post-surgery (see online suppl. Table S1).

We investigated patients with different operation techniques: $62.3 \%(n=175)$ underwent laparoscopic RYGB surgery, 22.1\% $(n=62)$ sleeve gastrectomy, and 15.6\% $(n=44)$ gastric banding. The metabolic and anthropomorphic effects of the three different methods are depicted in online supplementary Table S1: while the glucose levels of the 2-hour OGTT did not differ pre-surgery $(p=0.086)$, we found a significant difference in 2-hour glucose levels between gastric bypass $(3.2[2.4,4.2] \mathrm{mmol} / \mathrm{L})$, sleeve gastrectomy $(3.7[2.8,5.5] \mathrm{mmol} / \mathrm{L})$, and gastric banding $(4.6[3.8,6.1] \mathrm{mmol} / \mathrm{L})(p<0.0001)$. 


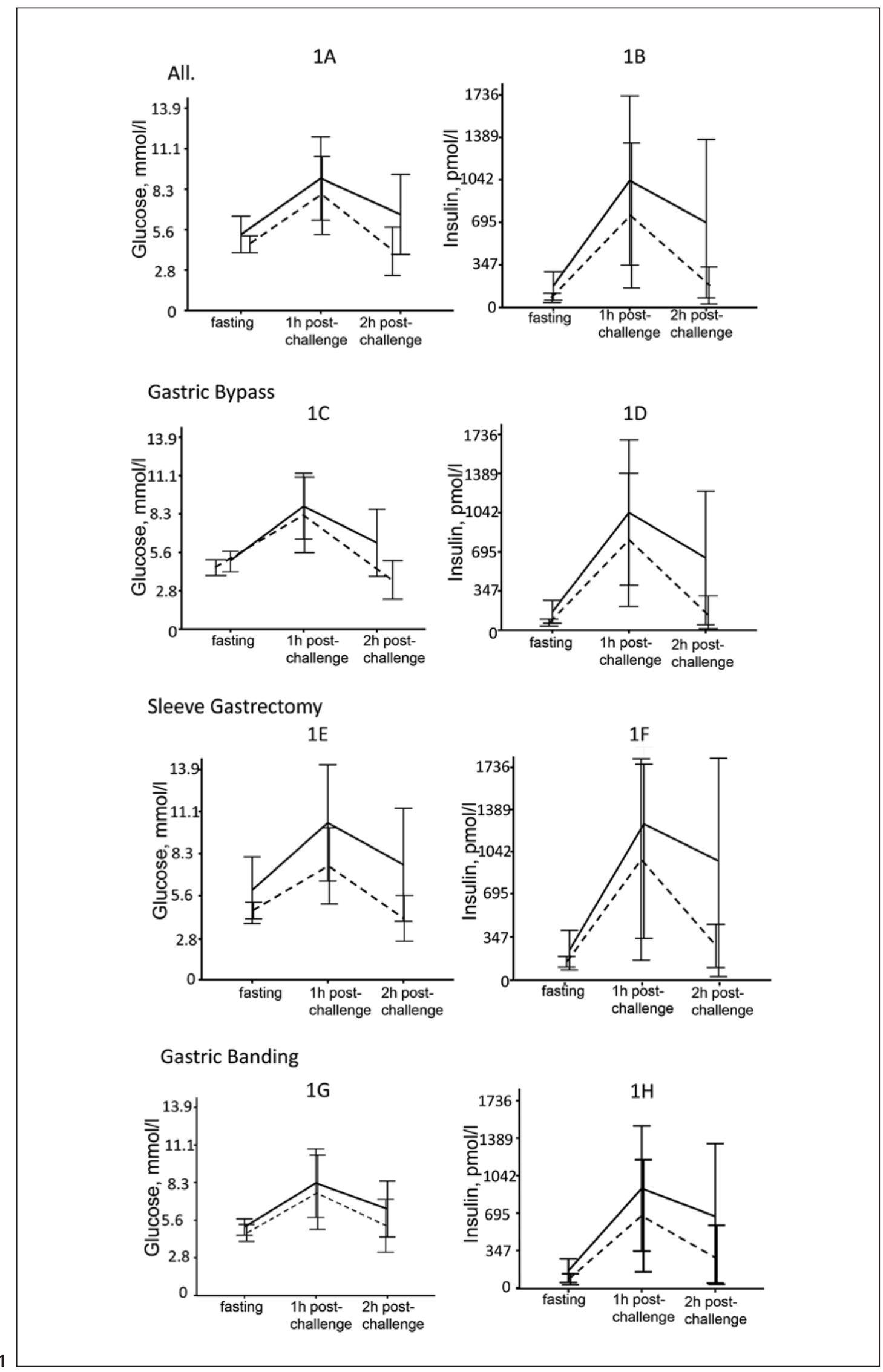

(For legend see next page.) 
Overall, hypoglycaemia was observed in $72(25.6 \%)$ of our 281 patients after OGTT postsurgery. None of our patients had fasting glucose values lower than $2.8 \mathrm{mmol} / \mathrm{L}$. Regarding the different surgical procedures, hypoglycaemia was observed in 57 patients $(32.6 \%)$ in the group that underwent gastric bypass surgery $(n=175)$ and in 14 patients $(22.6 \%)$ in the sleeve gastrectomy group $(n=62)$ (each post-surgery). By contrast, only 1 patient $(2.3 \%)$ presented with hypoglycaemia in the gastric banding group $(n=44)$.

In Figure $1 \mathrm{~A}-\mathrm{H}$, the different glucose and insulin levels pre- and post-surgery in all patients $(\mathrm{A}, \mathrm{B})$, as well as the three different operation techniques are shown: gastric bypass (C, D), sleeve gastrectomy (E, F), and gastric banding (G, H). As depicted in online supplementary Table S1, 2-hour glucose levels in patients post-gastric bypass were the lowest compared with other procedures: gastric bypass versus sleeve gastrectomy versus gastric banding: $3.2(2.4,4.2)$ versus $3.7(2.8,5.5)$ versus $4.6(3.8,6.1) \mathrm{mmol} / \mathrm{L}, p<0.001$.

Post-operative patients with and without hypoglycaemia did not differ in weight, BMI, waist circumference, or HOMA-IR. Online supplementary Figure S1 demonstrates glucose levels dynamically throughout the OGTT between patients with and without hypoglycaemia according to their type of operation.

\section{Differences in MMTT versus OGTT}

In 14 randomly selected subjects, we compared parameters of glucose metabolism between the OGTT and the MMTT. OGTT versus MMTT: fasting glucose: 4.5 (4.3-4.7) mmol/L versus 4.5 (4.3-5.1) $\mathrm{mmol} / \mathrm{L}, p=0.624$; 1-hour glucose: 6.7 (5.9-8.8) $\mathrm{mmol} / \mathrm{L}$ versus 7.4 (4.2-8.4) mmol/L, $p=0.433$; 2-hour glucose: 2.8 (2.3-4.7) mmol/L versus 2.3 (1.6-3.5) $\mathrm{mmol} / \mathrm{L}, p=0.022$; fasting insulin: 42 (33-60) pmol/L versus 39 (28-72) pmol/L, $p=0.701$; 1-hour insulin: 870 (297-1,211) pmol/L versus 680 (335-1,273) pmol/L, $p=0.878$; 2-hour insulin: 66 (38-285) pmol/L versus 46 (37-84) pmol/L, $p=0.929$.

\section{Symptoms of Hypoglycaemia}

Before surgery, none of the patients reported hypoglycaemic events or related symptoms during oGTT or in daily life. After surgery, 73 percent of all patients had symptoms of hypoglycaemia during OGTT. The most common symptoms were dizziness (81\%), weakness (79\%), and a feeling of general sickness (71\%). The majority of patients $(84 \%)$ with hypoglycaemia during OGTT reported that they had already had similar symptoms several times at home. Almost all of them reacted instinctively at home by eating carbohydrates and quickly feeling better afterwards. In addition, $10 \%$ of the patients who reported hypoglycaemic symptoms under daily life conditions did not show hypoglycaemia during the OGTT.

\section{Differences in Patients with or without Hypoglycaemia before $B S$}

Patients who presented with hypoglycaemia after BS differed from patients without hypoglycaemia after BS before undergoing surgery in age (39 [29 to 45] vs. 41 [37 to 51] years; $p=0.014$ ) and 2-hour post-challenge blood glucose values (5.5 [5.0 to 7.2] mmol/L vs. 6.9 [5.9 to 8.1] mmol/L; $p=0.001$ ). Weight, BMI, waist circumference, $\mathrm{HbA}_{1 \mathrm{c}}$, HOMA-IR, and insulin levels did not differ significantly for those with versus without hypoglycaemia (see online suppl. Table S2).

Fig. 1. Glucose and insulin levels in all patients (A, B), patients with gastric bypass (C, D), sleeve gastrectomy $(\mathbf{E}, \mathbf{F})$, and gastric banding $(\mathbf{G}, \mathbf{H})$. Data are shown in mean \pm standard deviation for simplification. The solid lines visualise the different dynamic changes of glucose and insulin levels pre- and the dashed lines postsurgery in between the different surgical procedures. 
Brix et al.: Hypoglycaemia after Bariatric Surgery

Differences in Patients with or without Hypoglycaemia after $B S$

Patients who presented with hypoglycaemia after BS differed from patients without hypoglycaemia after BS after surgery in weight (87 [79 to 102] vs. 90 [74 to 99] kg; $p=0.012$ ) and BMI (30 [27 to 35] vs. 32 [27 to 35]; $p=0.001$ ).

Regarding the OGTT, patients presenting with hypoglycaemia (2-hour post-challenge blood glucose: 2.3 [2.0 to 2.6] mmol/L vs. 3.9 [3.3 to 4.7] mmol/L; $p<0.001$ ) also had lower fasting blood glucose ( 4.3 [4.2 to 4.5] mmol/L vs. 4.5 [4.3 to 4.8] mmol/L; $p<0.001$ ), fasting insulin levels (49 [35 to 63] pmol/L vs. 56 [35 to 69] pmol/L; $p<0.001$ ), 2-hour post-challenge insulin levels (69 [35 to 97] pmol/L vs. 90 [49 to 167] pmol/L; $p<0.001$ ), and HOMA-IR (1.4 [0.9 to 1.7 ] vs. 1.5 [1.1 to 2.2 ]; $p<0.001$ ), but significantly higher 1-hour post-challenge insulin levels (729 [451 to 1,743$] \mathrm{pmol} / \mathrm{L}$ vs. 660 [319 to 1,000$] \mathrm{pmol} / \mathrm{L} ; p<0.001$ ) versus those without hypoglycaemia (see online suppl. Table S2).

Differences of the Change Pre-/Post-Surgery in Patients with and without Hypoglycaemia

Patients presenting with hypoglycaemia had greater decreases in body weight (43 [36 to 48 ] vs. 40 [33 to 48] $\mathrm{kg} ; p=0.013$ ) and BMI versus those patients without hypoglycaemia (14.7 [12.6 to 16.6] vs. 13.9 [11.3 to 17.1]; $p=0.037$ ). Regarding OGTT, patients with hypoglycaemia had a greater change in 2-hour post-challenge blood glucose values (3.6 [2.8 to 5.3] $\mathrm{mmol} / \mathrm{L}$ vs. 3.1 [1.7 to 4.4 ] mmol/L; $p=0.001$ ) and a smaller change in 1-hour post-challenge insulin levels (49 [-222 to 653] pmol/L vs. 42 [ -326 to 708] pmol/L; $p=0.004$ ) versus those patients without hypoglycaemia (see online suppl. Table S2).

Insulin Indices

The area under the curve (AUC) is significantly different before and after surgery in the whole group in glucose $\left(898.0 \pm 264.9 \mathrm{mmol} / \mathrm{L} / \mathrm{min}^{2}\right.$ vs. $707.6 \pm 166.5 \mathrm{mmol} / \mathrm{L} / \mathrm{min}^{2} ; p<$ $0.001)$ and insulin $\left(10,971[7,108\right.$ to 16,510$] \mu \mathrm{U} / \mathrm{mL} / \mathrm{min}^{2}$ vs. $5,664[3,587$ to 9,564$] \mu \mathrm{U} / \mathrm{mL} /$ $\left.\min ^{2} ; p<0.001\right)$. Patients with hypoglycaemia had significant differences in the glucose AUC compared with patients without after surgery $\left(672 \pm 159 \mathrm{mmol} / \mathrm{L} / \mathrm{min}^{2}\right.$ vs. $741 \pm 189$ $\left.\mathrm{mmol} / \mathrm{L} / \mathrm{min}^{2} ; p=0.007\right)$, but there were no differences before surgery $(855 \pm 217 \mathrm{mmol} / \mathrm{L} /$ $\min ^{2}$ vs. $913 \pm 278 \mathrm{mmol} / \mathrm{L} / \mathrm{min}^{2} ; p=0.145$ ). Regarding insulin AUC after surgery, again patients with hypoglycaemia had significant differences compared with patients without (7,091 [5,680 to 14,091$] \mu \mathrm{U} / \mathrm{mL} / \mathrm{min}^{2}$ vs. $5,386[3,441$ to 8,550$\left.] \mu \mathrm{U} / \mathrm{mL} / \mathrm{min}^{2} ; p<0.001\right)$, but no difference could be shown before surgery $\left(11,116[6,225\right.$ to 16,043$] \mu \mathrm{U} / \mathrm{mL} / \mathrm{min}^{2}$ vs. $10,887[7,284$ to 16,433$\left.] \mu \mathrm{U} / \mathrm{mL} / \mathrm{min}^{2} ; p=0.699\right)$.

Regarding the IGI, we could not find a significant difference before surgery - neither in the $\operatorname{IGI}_{60}(p=0.442)$ nor in the $\operatorname{IGI}_{120}(p=0.147)$ - in patients with compared to patients without hypoglycaemia after surgery. But we were able to detect a significant difference in the IGI after BS: patients with hypoglycaemia had a higher IGI I0 $_{0}$ compared to patients without hypoglycaemia $(263[149,417]$ vs. $160[67,319] ; p=0.002)$ and a lower $\operatorname{IGI}_{120}(-4[-23,1]$ vs. $8[-31,119] ; p=0.013)$.

\section{Discussion}

In this prospective study, we demonstrated that after BS, $25.6 \%$ of all our patients and $32.6 \%$ of those who underwent a gastric bypass procedure experience hypoglycaemia $(<2.8 \mathrm{mmol} / \mathrm{L})$ following an ingestion of $75 \mathrm{gms}$ of glucose. To date, BS is regarded as the most successful procedure for long-term weight loss and is recognised as a valid option for the treatment of morbid obesity itself but also for the treatment of type 2 diabetes in morbidly obese patients [12]. The overwhelming success of BS in treating morbid obesity 
might lead to a general neglect of potential and serious side effects. Marsk and colleagues [2] first demonstrated an increased likelihood of hospitalisation due to hypoglycaemia after BS. The absolute risk was only $0.2 \%$. The advantage of their study is the high number of patients, but the disadvantage of their register study is that patients were not challenged for hypoglycaemia. As Marsk and colleagues [2] looked at diagnostic codes at hospitalisation only, it is plausible - as Patti and Goldfine [5] proposed in their review - that the authors underestimated the prevalence of hypoglycaemia $[3,6]$. While episodes of severe hypoglycaemia are detected infrequently, the prevalence of low glucose values is relatively common and varies greatly, between 10.4 and 68\% [13-15]. This variability depends on the glycaemic load (from 30 gms of glucose in a test meal to 100 gms in OGTTs) and on the thresholds used to define hypoglycaemia. We have used $75 \mathrm{gms}$ in the OGTT and as threshold $<2.8 \mathrm{mmol} / \mathrm{L}$ defining post-challenge hypoglycaemia in order to exclude mild hypoglycaemic events. Pigeyre et al. [16] found a prevalence of hypoglycaemia of $10.4 \%$ after RYGB; however, they studied patients already after 1 year, whereas our follow-up study was done 2 years after BS. The difference in prevalence between the study of Pigeyre et al. [16] and ours might be explained by the fact that the frequency of hypoglycaemia gradually increases over time.

Our observation of a high rate of hypoglycaemic events after glucose load is in line with a previous small pilot study, in which 36 patients underwent a 100 gms OGTT at least 6 months after gastric bypass. In this study, Roslin and colleagues [14] demonstrated that $72 \%$ $(n=26)$ had evidence of reactive hypoglycaemia (defined as 2-hour post-glucose load blood glucose $\leq 3.3 \mathrm{mmol} / \mathrm{L}$ ). In contrast to our study, the sample size was small and one-third of the patients had diabetes before surgery [14]. In addition, a small pilot study published by Abhramsson et al. [17] using CGMS measurements in daily life for 3 days demonstrated that patients after gastric bypass as well as duodenal switch spent $2.9 \%$ time and $5.9 \%$ time, respectively, in hypoglycaemia. In contrast, Kefurt et al. [18] performed CGMS measurements in 40 patients and demonstrated a much higher frequency of hypoglycaemia (threshold used was $3.05 \mathrm{mmol} / \mathrm{L})$ under real-life conditions (75\% of the patients) 86 months after laparoscopic gastric bypass surgery.

Up to now, only a few studies have investigated hypoglycaemia after sleeve gastrectomy. There is increasing evidence that patients have an accelerated gastric emptying after sleeve gastrectomy $[19,20]$. Recently, an MRI study was performed to evaluate the motility of the gastric sleeve by using dynamic MRI. Preliminary results indicate accelerated gastric emptying due to faster peristaltic folds in the antrum [21]. Rapid gastric emptying may lead to an overexpression of gastric hormones, such as GLP-1 and PYY, which promote dumping syndrome $[22,23]$. However, hypoglycaemia after sleeve gastrectomy was far less frequently observed in our study than in the study by Tzovaras and colleagues [24], where the authors demonstrated that nearly one-third of all patients were suffering from dumping syndrome after laparoscopic sleeve gastrectomy.

Based on the results of our study, we were able to identify younger age at the time of surgery and a lower preoperative 2-hour post-challenge blood glucose as factors for an increased risk of postoperative hypoglycaemia. After surgery, hypoglycaemia was associated with lower fasting and lower 2-hour post-challenge insulin levels but significantly higher 1-hour post-challenge insulin levels. In addition, patients prone to hypoglycaemia had lower fasting blood glucose, HOMA-IR, and BMI than patients without hypoglycaemia. Regarding the change between pre- and post-surgery, patients with hypoglycaemia had smaller decreases in 1-hour post-challenge blood glucose values and 1-hour post-challenge insulin levels compared to patients without hypoglycaemia. Regarding 2-hour post-challenge blood glucose values, the decrease was larger in patients with hypoglycaemia compared with those without. Furthermore, we demonstrated differences in the AUC of 
Brix et al.: Hypoglycaemia after Bariatric Surgery

insulin levels, especially after BS in patients with compared to patients without hypoglycaemia.

In the study by Adams and colleagues [25], the risk of non-disease-related death increased by a factor of 1.85 compared with that in the control group, despite reduction in disease-related death after gastric bypass surgery. The increased rate of accidents remained unclear, but it cannot be excluded that unconsciousness or arrhythmia induced by hypoglycaemia are contributing factors. In 2007, Sjöström and colleagues [26] demonstrated, in the Swedish Obese Subjects (SOS) study, a significantly smaller number of myocardial infarctions in the group of patients who underwent BS compared with the control group (13 vs. 25 events), although in this study, operation techniques were used which are not used anymore today and only a minority of patients underwent gastric bypass surgery. But they also showed a higher number of sudden deaths due to cardiovascular condition in the surgery group (20 vs. 14 events) [26]. It is known that severe hypoglycaemia in patients with diabetes causes ST wave changes and leads to severe arrhythmias, such as QT prolongation [27]. Thus, arrhythmia due to hypoglycaemia might be a putative explanation for the increased rate of sudden death. The majority of patients undergoing BS are relatively young, favorably women, and have a rather low cardiovascular risk profile [26]. This might be the explanation why hypoglycaemia in those patients is not associated with a higher event rate compared with older patients with diabetes and a higher cardiovascular risk profile.

One limitation of our study is that an OGTT is an "experimental setting." However, the relatively high intake of carbohydrates in the OGTT (75 gms) might be representative of the daily consumption in this cohort. However, we have to admit that apart from soft drinks, patients usually do not consume 75 gms of glucose in only $15 \mathrm{~min}$. The change to a higher intake of carbohydrates could increase the risk of hypoglycaemia via post-challenge hyperinsulinaemia. Colles and colleagues [28] reported a reduction in percentage energy intake from fat and a concurrent increase in percentage energy intake from carbohydrates after BS. In addition, reports indicate that patients after gastric bypass surgery still consume large amounts of soft drinks; thus, in our cohort of patients, the OGTT might be representative [29]. Furthermore, our data are well in line with a recently published study by Lee et al. [30], where the authors sent questionnaires to patients at home after BS about the frequency of hypoglycaemia. The prevalence of hypoglycaemia is comparable to the prevalence achieved in our study. In contrast to this study, none of our patients had hypoglycaemia pre-surgery. To ascertain our findings, 14 randomly selected patients were additionally subjected to an MMTT. The postoperative insulin and glucose levels of these patients were absolutely comparable. However, the only difference we observed were even lower 2-hour post-stimulation glucose values during OGTT versus MMTT.

Since the aim of our study was to investigate the prevalence of hypoglycaemia and its relation to the surgical intervention, we could not reliably identify the reason for the occurrence of hypoglycaemia due to the lack of measurement of GLP-1 or other gastrointestinal hormones and studies on gastric transit time.

In conclusion, we were able to demonstrate the high frequency and thus the potential danger of hypoglycaemia following different procedures of BS in a large prospective, longitudinal study by OGTT. Based on our data, we recommend that every patient should routinely undergo an OGTT postoperatively after BS. Furthermore, the patients should be trained by a dietician to increase the number of meals per day and to reduce the amount of carbohydrates. In addition, we strongly recommend that, after $\mathrm{BS}$, every patient should be trained to recognise and treat hypoglycaemia as would patients with diabetes. 
Brix et al.: Hypoglycaemia after Bariatric Surgery

\section{Acknowledgments}

We especially thank Eike Lobesinner, Barbara Markl, and Heidemarie Tripolt, all professional nurses at the outpatient clinic of the Department of Medicine I, Krankenanstalt Rudolfstiftung, for the organization and conduct of the patients visits.

\section{Statement of Ethics}

The study was approved by the institutional ethics committee and all subjects gave written informed consent.

\section{Disclosure Statement}

The authors have no conflict of interest regarding this study.

\section{References}

1 WHO Obesity and Overweight. Fact sheet No. 311; May 2012: www.who.int/mediacentre/factsheets/fs311/ en/.

2 Marsk R, Jonas E, Rasmussen F, Näslund E. Nationwide cohort study of post-gastric bypass hypoglycaemia including 5,040 patients undergoing surgery for obesity in 1986-2006 in Sweden. Diabetologia. 2010 Nov; 53(11):2307-11.

3 Service GJ, Thompson GB, Service FJ, Andrews JC, Collazo-Clavell ML, Lloyd RV. Hyperinsulinemic hypoglycemia with nesidioblastosis after gastric-bypass surgery. N Engl J Med. 2005 Jul;353(3):249-54.

4 Schernthaner G, Brix JM, Kopp HP, Schernthaner GH. Cure of type 2 diabetes by metabolic surgery? A critical analysis of the evidence in 2010. Diabetes Care. 2011 May;34 Suppl 2:S355-60.

5 Patti ME, Goldfine AB. Hypoglycaemia following gastric bypass surgery-diabetes remission in the extreme? Diabetologia. 2010 Nov;53(11):2276-9.

6 Goldfine AB, Mun EC, Devine E, Bernier R, Baz-Hecht M, Jones DB, et al. Patients with neuroglycopenia after gastric bypass surgery have exaggerated incretin and insulin secretory responses to a mixed meal. J Clin Endocrinol Metab. 2007 Dec;92(12):4678-85.

7 Kim SH, Liu TC, Abbasi F, Lamendola C, Morton JM, Reaven GM, et al. Plasma glucose and insulin regulation is abnormal following gastric bypass surgery with or without neuroglycopenia. Obes Surg. 2009 Nov;19(11): $1550-6$.

8 Kopp HP, Krzyzanowska K, Schernthaner GH, Kriwanek S, Schernthaner G. Relationship of androgens to insulin resistance and chronic inflammation in morbidly obese premenopausal women: studies before and after vertical banded gastroplasty. Obes Surg. 2006 Sep;16(9):1214-20.

9 Workgroup on Hypoglycemia, American Diabetes Association. Defining and reporting hypoglycemia in diabetes: a report from the American Diabetes Association Workgroup on Hypoglycemia. Diabetes Care. 2005 May;28(5):1245-9.

10 Matthews DR, Hosker JP, Rudenski AS, Naylor BA, Treacher DF, Turner RC. Homeostasis model assessment: insulin resistance and beta-cell function from fasting plasma glucose and insulin concentrations in man. Diabetologia. 1985 Jul;28(7):412-9.

11 Hanson RL, Pratley RE, Bogardus C, Narayan KM, Roumain JM, Imperatore G, et al. Evaluation of simple indices of insulin sensitivity and insulin secretion for use in epidemiologic studies. Am J Epidemiol. 2000 Jan;151(2): 190-8.

12 Dixon JB, le Roux CW, Rubino F, Zimmet P. Bariatric surgery for type 2 diabetes. Lancet. 2012 Jun;379(9833): 2300-11.

13 Kellogg TA, Bantle JP, Leslie DB, Redmond JB, Slusarek B, Swan T, et al. Postgastric bypass hyperinsulinemic hypoglycemia syndrome: characterization and response to a modified diet. Surg Obes Relat Dis. 2008 Jul-Aug; 4(4):492-9.

14 Roslin M, Damani T, Oren J, Andrews R, Yatco E, Shah P. Abnormal glucose tolerance testing following gastric bypass demonstrates reactive hypoglycemia. Surg Endosc. 2011 Jun;25(6):1926-32.

15 Patti ME, Goldfine AB. Hypoglycemia after gastric bypass: the dark side of GLP-1. Gastroenterology. 2014 Mar; 146(3):605-8. 
Brix et al.: Hypoglycaemia after Bariatric Surgery

16 Pigeyre M, Vaurs C, Raverdy V, Hanaire H, Ritz P, Pattou F. Increased risk of OGTT-induced hypoglycemia after gastric bypass in severely obese patients with normal glucose tolerance. Surg Obes Relat Dis. 2015 May-Jun; 11(3):573-7.

17 Abrahamsson N, Edén Engström B, Sundbom M, Karlsson FA. Hypoglycemia in everyday life after gastric bypass and duodenal switch. Eur J Endocrinol. 2015 Jul;173(1):91-100.

18 Kefurt R, Langer FB, Schindler K, Shakeri-Leidenmühler S, Ludvik B, Prager G. Hypoglycemia after Roux-En-Y gastric bypass: detection rates of continuous glucose monitoring (CGM) versus mixed meal test. Surg Obes Relat Dis. 2015 May-Jun;11(3):564-9.

19 Melissas J, Daskalakis M, Koukouraki S, Askoxylakis I, Metaxari M, Dimitriadis E, et al. Sleeve gastrectomy-a "food limiting" operation. Obes Surg. 2008 Oct;18(10):1251-6.

20 Braghetto I, Davanzo C, Korn O, Csendes A, Valladares H, Herrera E, et al. Scintigraphic evaluation of gastric emptying in obese patients submitted to sleeve gastrectomy compared to normal subjects. Obes Surg. 2009 Nov;19(11):1515-21.

21 Baumann T, Kuesters S, Grueneberger J, Marjanovic G, Zimmermann L, Schaefer AO, et al. Time-resolved MRI after ingestion of liquids reveals motility changes after laparoscopic sleeve gastrectomy-preliminary results. Obes Surg. 2011 Jan;21(1):95-101.

22 Valderas JP, Irribarra V, Rubio L, Boza C, Escalona M, Liberona Y, Matamala A, Maiz A. Effects of sleeve gastrectomy and medical treatment for obesity on glucagon-like peptide 1 levels and glucose homeostasis in non-diabetic subjects. Obes Surg. 2011 Jul;21(7):902-9.

23 Valderas JP, Irribarra V, Boza C, de la Cruz R, Liberona Y, Acosta AM, et al. Medical and surgical treatments for obesity have opposite effects on peptide YY and appetite: a prospective study controlled for weight loss. J Clin Endocrinol Metab. 2010 Mar;95(3):1069-75.

24 Tzovaras G, Papamargaritis D, Sioka E, Zachari E, Baloyiannis I, Zacharoulis D, et al. Symptoms suggestive of dumping syndrome after provocation in patients after laparoscopic sleeve gastrectomy. Obes Surg. 2012 Jan; 22(1):23-8.

25 Adams TD, Gress RE, Smith SC, Halverson RC, Simper SC, Rosamond WD, et al. Long-term mortality after gastric bypass surgery. N Engl J Med. 2007 Aug;357(8):753-61.

26 Sjöström L, Narbro K, Sjöström CD, Karason K, Larsson B, Wedel H, Lystig T, Sullivan M, Bouchard C, Carlsson B, Bengtsson C, Dahlgren S, Gummesson A, Jacobson P, Karlsson J, Lindroos AK, Lönroth H, Näslund I, Olbers T, Stenlöf K, Torgerson J, Agren G, Carlsson LM. Effects of bariatric surgery on mortality in Swedish obese subjects. N Engl J Med. 2007;357(8):741-52.

27 Frier BM, Schernthaner G, Heller SR. Hypoglycemia and cardiovascular risks. Diabetes Care. 2011 May;34 Suppl 2:S132-7.

28 Colles SL, Dixon JB, O’Brien PE. Hunger control and regular physical activity facilitate weight loss after laparoscopic adjustable gastric banding. Obes Surg. 2008 Jul;18(7):833-40.

29 Ernst B, Thurnheer M, Wilms B, Schultes B. Differential changes in dietary habits after gastric bypass versus gastric banding operations. Obes Surg. 2009 Mar;19(3):274-80.

30 Lee CJ, Clark JM, Schweitzer M, Magnuson T, Steele K, Koerner O, et al. Prevalence of and risk factors for hypoglycemic symptoms after gastric bypass and sleeve gastrectomy. Obesity (Silver Spring). 2015 May;23(5): 1079-84. 